\title{
First-principles study of magnetization relaxation enhancement and spin transfer in thin magnetic films
}

\author{
Maciej Zwierzycki, ${ }^{1}, *$ Yaroslav Tserkovnyak, ${ }^{2}$ Paul J. Kelly, ${ }^{1}$ Arne Brataas, ${ }^{3}$ and Gerrit E. W. Bauer ${ }^{4}$ \\ ${ }^{1}$ Faculty of Science and Technology and MESA ${ }^{+}$Research Institute, University of Twente, 7500 AE Enschede, The Netherlands \\ ${ }^{2}$ Lyman Laboratory of Physics, Harvard University, Cambridge, Massachusetts 02138, USA \\ ${ }^{3}$ Department of Physics, Norwegian University of Science and Technology, N-7491 Trondheim, Norway \\ ${ }^{4}$ Kavli Institute of NanoScience, Delft University of Technology, 2628 CJ Delft, The Netherlands \\ (Received 3 February 2004; revised manuscript received 3 December 2004; published 28 February 2005)
}

\begin{abstract}
The interface-induced magnetization damping of thin ferromagnetic films in contact with normal-metal layers is calculated from first principles for clean and disordered $\mathrm{Fe} / \mathrm{Au}$ and $\mathrm{Co} / \mathrm{Cu}$ interfaces. Interference effects arising from coherent scattering turn out to be very small, consistent with a very small magnetic coherence length. Because the mixing conductances which govern the spin transfer are to a good approximation real-valued, the spin pumping can be described by an increased Gilbert damping factor but an unmodified gyromagnetic ratio. The results also confirm that the spin-current-induced magnetization torque is an interface effect.
\end{abstract}

DOI: 10.1103/PhysRevB.71.064420

PACS number(s): 75.70.Cn, 76.50.+g, 71.15.Ap, 72.25.Mk

\section{INTRODUCTION}

The local magnetization dynamics in a bulk ferromagnet are usually well described by a phenomenological model formulated in terms of three parameters: $\mathbf{H}_{\text {eff }}$, an effective magnetic field; $\gamma$, a gyromagnetic ratio; and $\alpha$, a Gilbert-damping constant. The field $\mathbf{H}_{\text {eff }}$ is a sum of contributions from externally applied fields, crystal anisotropy, shape-dependent dipolar interactions, and exchange interactions which govern ferromagnetic spin-wave spectral characteristics. $\gamma$ is the ratio of the total magnetic moment and the angular momentum of the electrons in the ferromagnet; in $3 d$ transition-metal ferromagnets, such as $\mathrm{Fe}$ and $\mathrm{Co}$, it is close to the freeelectron value $\gamma \approx 2 \mu_{B} / \hbar$. The Gilbert-damping constant $\alpha$ parametrizes the viscous damping of an excited magnetization to the (locally) lowest-energy configuration. Its value differs considerably for various materials and also depends on the temperature and on the impurity/defect composition of a given sample. The motion of the magnetization-direction unit vector $\mathbf{m}$ is determined by the phenomenological Landau-Lifshitz-Gilbert (LLG) equation: ${ }^{1}$

$$
\frac{d \mathbf{m}}{d t}=-\gamma \mathbf{m} \times \mathbf{H}_{\text {eff }}+\alpha \mathbf{m} \times \frac{d \mathbf{m}}{d t} .
$$

The magnetization dynamics of small monodomain ferromagnets are well described by the LLG equation (1) down to the micron scale. New effects may play a role on the submicron scale, however. The magnetization dynamics is no longer a highly coherent process because interface and surface roughness are relatively more important in small samples. Many-magnon processes can then acquire a sizeable spectral weight ${ }^{2}$ and are observable as, e.g., an increased line width of the ferromagnetic resonance (FMR). ${ }^{3}$ Another source of additional FMR broadening is nonlocal, depending on the environment into which the ferromagnet is embedded: a time-dependent ferromagnetic order parameter pumps spin currents that carry angular momentum (and energy) into adjacent conducting materials. ${ }^{4,5}$ This angular-momentum loss, in turn, is equivalent to an additional damping torque on the magnetization. ${ }^{6}$

The spin-pumping concept for the magnetization dynamics of nanostructures has far-reaching consequences. It gives rise to an enhanced Gilbert damping of magnetic films in contact with conducting media, ${ }^{4}$ may be employed as an FMR-operated spin battery, ${ }^{7}$ and explains a dynamic exchange coupling in magnetic bilayers, ${ }^{8,9}$ as well as a dynamic stiffness against current-induced magnetization reversal. ${ }^{10}$ The analysis of experimental FMR probes of the magnetization dynamics in single films ${ }^{5}$ and magnetic bilayers ${ }^{8}$ relied on phenomenological models of the electronic structure. Here we show how these assumptions can be relaxed by using instead scattering matrices calculated from first principles which take into account the detailed atomic and electronic structure of the materials under study.

An early phenomenological treatment of the nonlocality of the magnetization dynamics in hybrid normal-metal/ ferromagnet $(N / F)$ structures was given by Silsbee et al. ${ }^{11}$ Recently, Šimánek et al. ${ }^{12}$ pointed out that time-dependent linear-response theory could be used to calculate the spin flows generated by a ferromagnet with a time-varying magnetization in contact with a nonmagnetic conductor, as an alternative to the scattering-theory approach of Tserkovnyak et al. ${ }^{4}$ In spite of the different starting point, complete agreement between the two methods was demonstrated ${ }^{13}$ for the simple case of a $\delta$-function magnetic layer embedded in a free-electron gas. In addition, it was argued in Refs. 12 and 13 that the electron-electron interactions can considerably enhance the spin currents into normal metals with large Stoner-enhancement factors. The linear-response framework has also been used to calculate the enhanced Gilbert damping of finite-thickness ferromagnetic films. ${ }^{14}$ It was argued there that ultra-thin films display oscillatory damping (as a function of thickness) due to quantum-size effects. In the following, we show that these quantum-interference effects are greatly overestimated by the ballistic free-electron band model and do not survive when realistic transition-metal 
band structures are used. By calculating from first principles the scattering matrix entering the spin-pumping theory, ${ }^{4}$ we show that quantum-size oscillations are much smaller than those reported in Ref. 14, especially if even small amounts of disorder are introduced. We also find that the additional term in the ferromagnetic equation of motion is of the Gilbertdamping form, with only a very small correction to the gyromagnetic ratio (the same conclusion can also be drawn from previous work ${ }^{15}$ ). Furthermore, the electron-electron interaction effects discussed by Šimánek ${ }^{13}$ are taken into account in the exchange-correlation potential which we calculate self-consistently within the local spin density approximation (LSDA) of density-functional theory; they do not give rise to enhanced Gilbert damping in our picture. The electronic structure of the normal metal enters the expressions for the Gilbert damping not via the Fermi energy density of states but via the Sharvin conductance. Finally, our results confirm that the spin-current-induced magnetization torque $^{16}$ is an interface effect, which was earlier taken for granted $^{17,18}$ and analyzed in detail in Ref. 19.

This article is organized as follows. The general theory of spin pumping and its consequences for the dynamics of the precessing ferromagnet are reviewed in Sec. II. In Sec. III we describe the first-principles methods used to obtain the results presented and discussed in Sec. IV. A comparison with results based on a free-electron model is made in the Appendix and conclusions are drawn in Sec. V.

\section{THEORY}

We first consider a ferromagnetic film of thickness $d$ connected to two perfect nonmagnetic reservoirs by two leads which support well-defined scattering states. The electrons incident on the ferromagnet from a lead are distributed according to the Fermi-Dirac statistics of the respective reservoir, whereas the probability that an electron leaving the ferromagnet returns there with finite spin (or phase) memory is vanishingly small. Such perfect spin sinks can be realized experimentally by attaching leads to the ferromagnetic film in the form of point contacts with dimensions smaller than the electron mean free path. ${ }^{20}$ Alternatively, a normal conductor with a very high spin-flip to momentum scatteringrate ratio (as could be provided by heavy impurities with large spin-orbit interaction in a light metal or a heavy metal with phonon or defect scatterers) can serve as a good spin sink. ${ }^{5}$

Coherent motion of the magnetization, whose direction is given by the unit vector $\mathbf{m}(t)$, leads to the emission of a spin current

$$
\mathbf{I}_{s}=\frac{\hbar}{4 \pi}\left(\operatorname{Re} A^{\uparrow \downarrow} \mathbf{m} \times \frac{d \mathbf{m}}{d t}+\operatorname{Im} A^{\uparrow \downarrow} \frac{d \mathbf{m}}{d t}\right)
$$

per unit area of the contact into each normal-metal layer, ${ }^{4}$ which we will here assume is then fully absorbed by the spin sinks (reservoirs). ${ }^{5}$ The complex spin-pumping conductance $^{21}$

$$
A^{\uparrow \downarrow}=g_{\uparrow \downarrow}^{r}-g_{\uparrow \downarrow}^{t}
$$

is the difference between the reflection $\left(g_{\uparrow \downarrow}^{r}\right)$ and transmission $\left(g_{\uparrow \downarrow}^{t}\right)$ mixing conductances (per unit contact area) which are defined in terms of the spin-dependent reflection and transmission matrices of the ferromagnetic film as ${ }^{18,22}$

$$
\begin{gathered}
g_{\uparrow \downarrow}^{r}=S^{-1} \sum_{m n}\left(\delta_{m n}-r_{m n}^{\uparrow} r_{m n}^{\downarrow \star}\right), \\
g_{\uparrow \downarrow}^{t}=S^{-1} \sum_{m n} t_{m n}^{\prime \uparrow} t_{m n}^{\prime \downarrow \star} .
\end{gathered}
$$

Here, $S$ is the $F / N$ contact area, and $m$ and $n$ denote scattering states at the Fermi energy of the normal-metal leads. For spin-pumping into one of the normal-metal layers, $g_{\uparrow \downarrow}^{r}$ is expressed in terms of the amplitude $r_{m n}^{\sigma}$ for an incoming electron in state $m$ of the normal metal to be reflected at the interface with the magnetic film into the outgoing state $n$, while $g_{\uparrow \downarrow}^{t}$ is expressed in terms of the amplitude $t_{m n}^{\prime \sigma}$ for an incoming electron from the other $N$ layer to be transmitted across the ferromagnet into the outgoing state $n$. The total angular-momentum loss of the ferromagnet is given by a sum of contributions (2) from the two leads, characterized by two spin-pumping parameters $A_{1}^{\uparrow \downarrow}$ and $A_{2}^{\uparrow \downarrow}$. As explained in Ref. 4, adding this source of spin angular-momentum current to the right-hand side of Eq. (1) leads to a new LLG equation for the monodomain thin film with saturation magnetization $M_{s}$ embedded in the nonmagnetic conducting medium, with the modified constants $\alpha_{\text {eff }}$ and $\gamma_{\text {eff: }}$ :

$$
\begin{gathered}
\frac{1}{\gamma_{\text {eff }}}=\frac{1}{\gamma}\left[1-\frac{\hbar \gamma}{4 \pi M_{s} d} \operatorname{Im}\left(A_{1}^{\uparrow \downarrow}+A_{2}^{\uparrow \downarrow}\right),\right. \\
\alpha_{\text {eff }}=\frac{\gamma_{\text {eff }}}{\gamma}\left[\alpha+\frac{\hbar \gamma}{4 \pi M_{s} d} \operatorname{Re}\left(A_{1}^{\uparrow \downarrow}+A_{2}^{\uparrow \downarrow}\right)\right] .
\end{gathered}
$$

It can be easily shown ${ }^{4}$ that the real part of $A^{\uparrow \downarrow}$ is always non-negative so that the correction to the damping is always positive. The reader is referred to Sec. IV for a discussion of the absolute and relative values of $g_{\uparrow \downarrow}^{r}$ and $g_{\uparrow \downarrow}^{t}$. Anticipating these results, we note here that in typical situations $g_{\uparrow \downarrow}^{t}$ and $\operatorname{Im} g_{\uparrow \downarrow}^{r}$ (and thus $\operatorname{Im} A^{\uparrow \downarrow}$ ) are negligible so that the only effect of the spin pumping is to make an additional contribution to the Gilbert-damping parameter. We shall therefore assume for the rest of the current section that $g_{\uparrow \downarrow}^{t} \ll g_{\uparrow \downarrow}^{r}$ with the latter quantity being essentially an interface property.

Equation (2) was derived for an $N / F / N$ structure connected to perfectly equilibrated reservoirs. ${ }^{4,5}$ By using this geometry, the finiteness of the Sharvin conductances is automatically included. ${ }^{23}$ To apply calculated mixing conductances to the discussion of spin transport in diffuse systems which are not ideal spin sinks, the "bare" conductance (4) has to be corrected ${ }^{24}$ for the corresponding "spurious" Sharvin resistance as discussed in Ref. 25. Additionally, a nonvanishing backflow and reabsorption of the spins emitted by the ferromagnet has to be taken into account. The latter can be achieved by considering the diffusion equation for the spin accumulation in the normal lead with Eq. (2) providing the boundary condition (see Ref. 5). This leads to an effec- 
tive (complex) conductance $\widetilde{A}^{\uparrow \downarrow}$ (for either interface) entering Eqns. (6) and (7) where

$$
\frac{1}{\tilde{A}^{\uparrow \downarrow}}=\frac{1}{g_{\uparrow \downarrow}^{r}}-\frac{1}{2 g_{N}^{\mathrm{Sh}}}+\frac{2 e^{2}}{h} \cdot \frac{R_{\mathrm{SD}}}{\tanh \left(L / \lambda_{\mathrm{SD}}\right)},
$$

$g_{N}^{\text {Sh }}$ is the Sharvin conductance of the normal-metal layer, given by the number of the transverse channels per spin and unit area of the interface, ${ }^{25} R_{\mathrm{SD}}=\lambda_{\mathrm{SD}} / \sigma$ is the unit-area resistance of the normal-metal film with conductivity $\sigma / 2$ (per spin) and thickness $\lambda_{\mathrm{SD}}$, the spin-diffusion length; and $L$ is the actual thickness of the normal-metal layer. The last term on the right-hand side of Eq. (8) accounts for impurity, defect, or phonon scattering in the normal metal. (Scattering in the ferromagnet on length scales longer than the transverse spin-coherence length does not modify the result.) When spin-flip scattering in the $N$ layer vanishes, $\lambda_{\mathrm{SD}} \rightarrow \infty, \widetilde{A}^{\uparrow \downarrow}$ $\rightarrow 0$ (i.e., the backflow spin-current completely cancels the pumping effect) and the magnetization dynamics is not modified at all.

A similar analysis can be applied to magnetic damping in more complex multilayer systems. ${ }^{5,8}$ For example, in an $F / N / F$ structure the presence of two ferromagnetic layers can make damping possible for each individual layer even in the absence of spin-flip relaxation in the system. In this case, each magnetic layer acts as the sink for the spin current pumped by the other layer. If the structure is weakly excited from a collinear equilibrium state, and the individual ferromagnetic resonances are well separated, then a different effective conductance enters Eqs. (6) and (7). Instead of the $\operatorname{sum} A_{1}^{\uparrow \downarrow}+A_{2}^{\uparrow \downarrow}$, for the two magnetic films, the quantity $\widetilde{A}_{F / N / F}^{\uparrow \downarrow}$ with

$$
\frac{1}{\widetilde{A}_{F / N / F}^{\uparrow \downarrow}}=\frac{1}{g_{\uparrow \downarrow}^{1 r}}+\frac{1}{g_{\uparrow \downarrow}^{2 r}}-\frac{1}{g_{N}^{\mathrm{Sh}}}+\frac{2 e^{2}}{h} \cdot \frac{L}{\sigma}
$$

defined for the globally diffuse system should be used, where $g_{\uparrow \downarrow}^{i r}$ is the mixing conductance for the $i$ th $F / N$ interface. Equation (9) can be intuitively interpreted in terms of resistances in series: in order to be absorbed, the spin current must be pumped through the first $F / N$ interface $\left(g_{\uparrow \downarrow}^{1 r}\right.$ renormalized by $\left.2 g_{N}^{\mathrm{Sh}}\right)$, propagate across the normal layer $(L / \sigma$ term), and enter the second ferromagnet through the other interface $\left(g_{\uparrow \downarrow}^{2 r}\right.$ renormalized by $\left.2 g_{N}^{\mathrm{Sh}}\right)$. The formula for $\tilde{A}_{F / N / F}^{\uparrow \downarrow}$ can be straightforwardly derived using the spin-diffusion approach of Ref. 5. It is worthwhile pointing out that it remains correct for nondiffusive normal metal spacers $(\sigma \rightarrow \infty)$ if the interface disorder is sufficient to suppress any quantum-size effects (see Ref. 25).

The effect of spin-dependent scattering on the time evolution of the magnetic order parameter is therefore mostly governed by three parameters: the reflection and transmission mixing conductances of the ferromagnetic layer, $g_{\uparrow 1}^{r}$ and $g_{\uparrow \downarrow}^{t}$, and the Sharvin conductance of the normal metal, $g_{N}^{\mathrm{Sh}}$. We noted before ${ }^{4}$ that these quantities are in principle accessible to $a b$ initio electronic-structure calculations. ${ }^{15,26,27}$ In the following we demonstrate this by studying two representative $N / F$ material combinations: $\mathrm{Au} / \mathrm{Fe}(001)$ and
$\mathrm{Cu} / \mathrm{Co}(111)$, the former routinely used by the Simon-Fraser group $^{28-30}$ and the latter by the Cornell group. ${ }^{20,31}$

\section{FIRST-PRINCIPLES METHOD}

Parameter-free calculations of transmission and reflection coefficients were performed using the local spin density approximation (LSDA) of density-functional theory (DFT) in a two-step procedure. In the first step, the self-consistent electronic structure (spin densities and potentials) of the system was determined using the layer TB-LMTO (tight-binding linear muffin-tin orbital) surface Green's function (SGF) method in the atomic-sphere approximation (ASA). ${ }^{32}$ The exchange-correlation potential in the Perdew-Zunger ${ }^{33}$ parametrization was used. The atomic-sphere (AS) potentials of four monolayers on either side of the magnetic layer (or interface) were iterated to self-consistency while the potentials of more distant layers were held fixed at their bulk values. Because both of the systems we consider, $\mathrm{Au} / \mathrm{Fe}(001)$ and $\mathrm{Cu} / \mathrm{Co}(111)$, are nearly ideally lattice matched, common lattice constants were assumed for both metals of a given structure: $a_{\mathrm{Cu} / \mathrm{Co}}=3.549 \AA$ and $a_{\mathrm{Au} / \mathrm{Fe}}=\sqrt{2} \times 2.866=4.053 \AA$. In the second step, the AS potentials serve as inputs to calculate scattering coefficients using a recently developed scheme based on TB-MTOs. ${ }^{26,34,35}$ Disorder is modeled by allowing a number of interface layers to consist of $N_{x} F_{1-x}$ alloy which is modeled using repeated lateral supercells. Because a minimal basis set of $s, p$ and $d$ orbitals is used, we are able to treat lateral supercells containing as many as 200 atoms in which the two types of atoms are distributed at random in the appropriate concentration. For disordered interfaces, the AS potentials were calculated self-consistently using the layer CPA approximation in which each layer can have a different alloy composition. ${ }^{32}$

Little is known from experiment about the atomic structure of metallic interfaces. We model "dirty" interfaces with one (for $N / F / N$ systems) or two (for single $N / F$ interfaces) atomic layers of a 50\%-50\% alloy. Such a model is probably reasonable for $\mathrm{Cu}_{\mathrm{fcc}} / \mathrm{Co}_{\mathrm{fcc}}$ because of the nearly perfect lattice match and structural compatibility. The situation is, however, more complicated for $\mathrm{Au}_{\mathrm{fcc}} / \mathrm{Fe}_{\mathrm{bcc}}$ because of the large difference in AS sizes for $\mathrm{Au}$ and Fe with Wigner-Seitz radii of 2.99 and 2.67 Bohr atomic units, respectively. We have assumed here that the disorder is only substitutional and that the diffused atoms occupy the AS of the same size as that of the host element. In the $\mathrm{Au} / \mathrm{Fe} / \mathrm{Au}$ case, where the alloy is only 1 atomic monolayer (ML) thick, we assume that the Fe atoms diffuse into $\mathrm{Au}$. While the validity of this model can be questioned, the insensitivity of the final results to the details of the disorder (e.g., one versus two monolayers of alloy) indicate that this is not a critical issue. The layerresolved magnetic moments for single interfaces are given in Table I. They agree well with values reported previously in the literature. ${ }^{36-38}$

The two-dimensional Brillouin zone (2D BZ) summation required to calculate the mixing conductances using Eqs. (4) and (5) was performed using $k_{\|}$-mesh densities corresponding to $10^{4}$ points in the $2 \mathrm{D} \mathrm{BZ}$ of a $1 \times 1$ interface unit cell. The uncertainties resulting from this BZ summation and 
TABLE I. Layer-resolved magnetic moments in Bohr magnetons for single $N / F$ interfaces $(N=\mathrm{Au}, \mathrm{Cu} ; F=\mathrm{Fe}, \mathrm{Co})$.

\begin{tabular}{|c|c|c|c|c|}
\hline \multirow{2}{*}{$\begin{array}{l}N / F \\
\text { Layer }\end{array}$} & \multicolumn{2}{|c|}{$\mathrm{Au} / \mathrm{Fe}$} & \multicolumn{2}{|c|}{$\mathrm{Cu} / \mathrm{Co}$} \\
\hline & Clean & Dirty & Clean & Dirty \\
\hline$m_{N}($ bulk $)$ & 0.000 & 0.000 & 0.000 & 0.000 \\
\hline$m_{N}($ int-4) & 0.000 & 0.000 & 0.001 & 0.000 \\
\hline$m_{N}($ int -3$)$ & 0.001 & -0.003 & -0.000 & -0.003 \\
\hline$m_{N}($ int -2$)$ & -0.002 & 0.010 & -0.004 & -0.003 \\
\hline$m_{N}($ int-1) & 0.064 & 0.026 & 0.006 & 0.010 \\
\hline$m_{F}($ int-1) & & 2.742 & & 1.410 \\
\hline$m_{N}($ int +1$)$ & & 0.128 & & 0.036 \\
\hline$m_{F}(\mathrm{int}+1)$ & 2.687 & 2.691 & 1.545 & 1.540 \\
\hline$m_{F}(\mathrm{int}+2)$ & 2.336 & 2.396 & 1.635 & 1.596 \\
\hline$m_{F}(\mathrm{int}+3)$ & 2.325 & 2.363 & 1.621 & 1.627 \\
\hline$m_{F}(\mathrm{int}+4)$ & 2.238 & 2.282 & 1.627 & 1.624 \\
\hline$m_{F}($ bulk $)$ & 2.210 & 2.210 & 1.622 & 1.622 \\
\hline
\end{tabular}

from impurity ensemble averaging are of the order of a few times $10^{12} \Omega^{-1} \mathrm{~m}^{-2}$, which is smaller than the size of the symbols used in the figures.

\section{RESULTS AND DISCUSSION}

Figures 1-4 show how $G_{\uparrow \downarrow}^{r}=\left(e^{2} / h\right) g_{\uparrow \downarrow}^{r}$ and $G_{\uparrow \downarrow}^{t}$ $=\left(e^{2} / h\right) g_{\uparrow \downarrow}^{t}$ depend on the thickness $d$ of the magnetic layer (measured in atomic layers) for specular ( $\vec{k}_{\|}$-preserving) $\mathrm{Au} /$ $\mathrm{Fe} / \mathrm{Au}(001)$ and $\mathrm{Cu} / \mathrm{Co} / \mathrm{Cu}(111)$ systems. Both quantities exhibit oscillatory behavior with, however, noticeably different periods and amplitudes. The values of both $G_{\uparrow \downarrow}^{r}$ and $G_{\uparrow \downarrow}^{t}$ are determined by two factors: the matching of the normal metal and ferromagnetic metal states at the interface (described by the scattering coefficients of the single interface) and the phases accumulated by electrons on their passage through

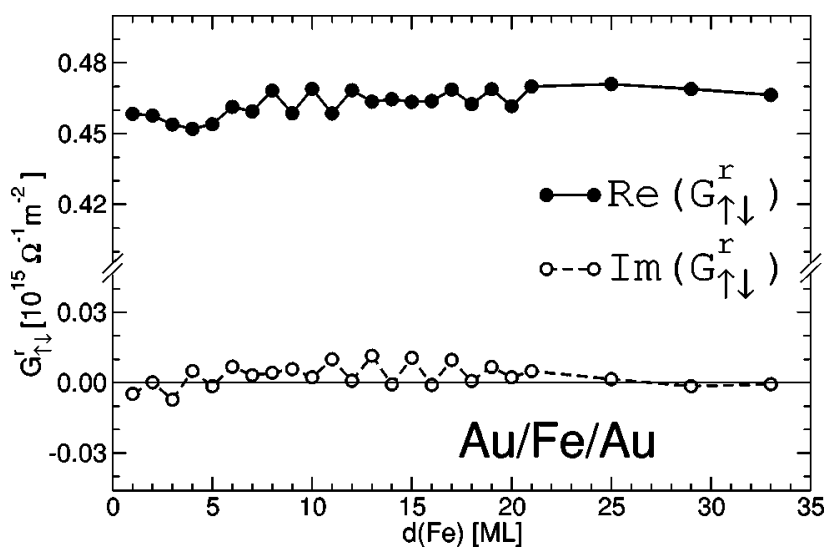

FIG. 1. Reflection spin-mixing conductance (per unit area) of a $\mathrm{Au} / \mathrm{Fe} / \mathrm{Au}(001)$ trilayer with perfect interfaces as a function of the thickness $d$ of the Fe layer. In this and subsequent plots, mixing conductances expressed in terms of number of conduction channels per unit area are converted to $\Omega^{-1} m^{-2}$ using the conductance quantum $e^{2} / h$, i.e., $G_{\uparrow \downarrow}=\left(e^{2} / h\right) g_{\uparrow \downarrow}$.

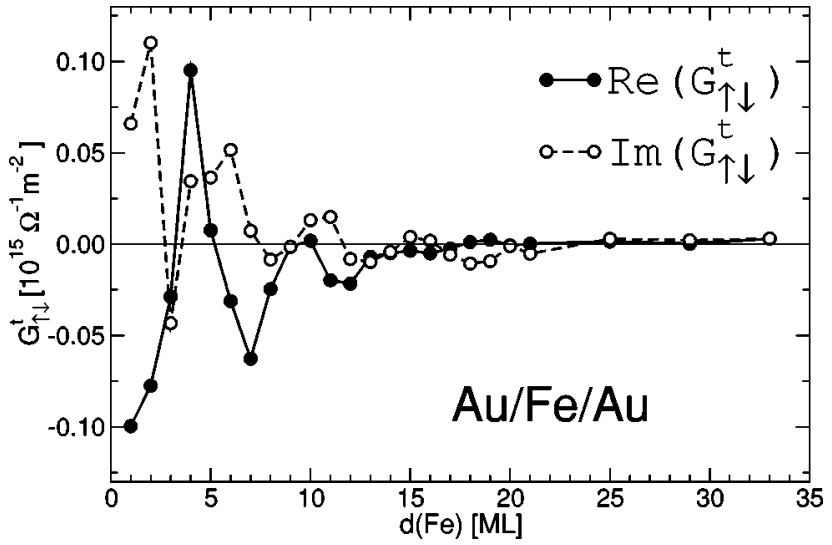

FIG. 2. Transmission spin-mixing conductance of a $\mathrm{Au} / \mathrm{Fe} / \mathrm{Au}$ (001) trilayer with perfect interfaces as a function of the thickness $d$ of the Fe layer.

the magnetic layer (quantum-size effect). The first factor determines the amplitudes of the oscillations and (for $G_{\uparrow \downarrow}^{r}$ ) the asymptotic values, while the second is responsible for the observed periodicity. In order to better understand this, it is instructive to interpret the transmission and reflection coefficients of the finite-size magnetic layer in terms of multiple scattering at the interfaces. We first note that both $\mathrm{Cu}$ and $\mathrm{Au}$ have only one left- and one right-going state at the Fermi level for each value of $\vec{k}_{\|}$and spin so that the summations in Eqs. (4) and (5) reduce to integrations over the 2D BZ involving the complex-valued functions $r^{\sigma}\left(\vec{k}_{\|}\right)$and $t^{\sigma}\left(\vec{k}_{\|}\right)$. Retaining only lowest-order thickness-dependent terms, dropping explicit reference to $\vec{k}_{\|}$and to the primes on $t^{\prime}$, we then have

$$
\begin{gathered}
t^{\sigma} \approx t_{F \rightarrow N}^{\sigma} \Lambda^{\sigma} t_{N \rightarrow F}^{\sigma} \\
r^{\sigma} \approx r_{N \rightarrow N}^{\sigma}+t_{F \rightarrow N}^{\sigma} \Lambda^{\sigma} r_{F \rightarrow F}^{\sigma} \Lambda^{\sigma} t_{N \rightarrow F}^{\sigma}
\end{gathered}
$$

where $t_{N \rightarrow F}^{\sigma}=\left(t_{1}^{\sigma}, \ldots, t_{n}^{\sigma}\right)^{T}$ is a vector of transmission coefficients between a single propagating state in the normal metal and a set of states in the ferromagnet, $\Lambda^{\sigma}$ is a diagonal matrix

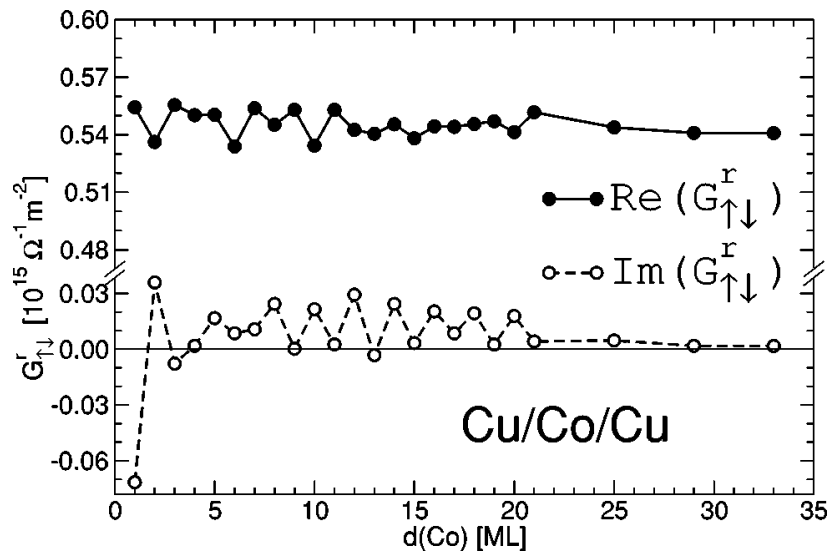

FIG. 3. Reflection spin-mixing conductance of a $\mathrm{Cu} / \mathrm{Co} / \mathrm{Cu}$ (111) trilayer with perfect interfaces as a function of the thickness $d$ of the Co layer. 


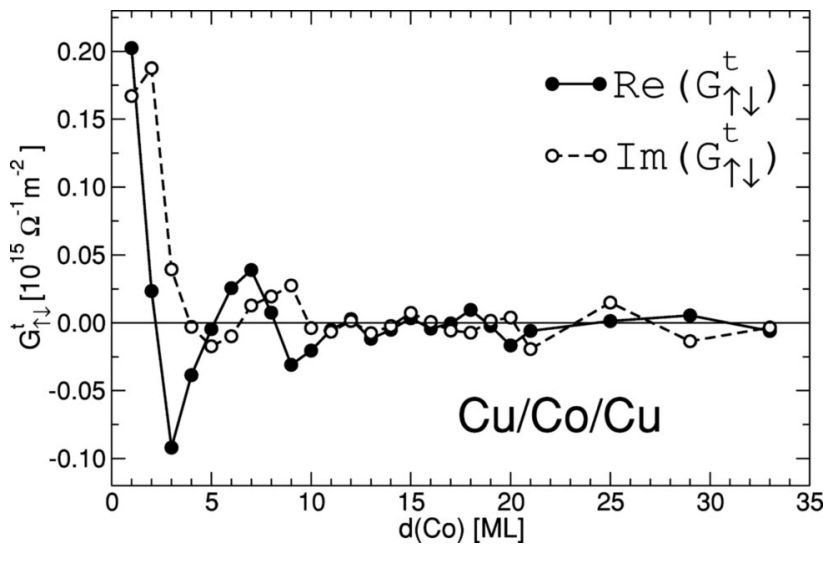

FIG. 4. Transmission spin-mixing conductance of a $\mathrm{Cu} / \mathrm{Co} / \mathrm{Cu}$ (111) trilayer with perfect interfaces as a function of the thickness $d$ of the Co layer.

of phase factors $e^{i k_{\perp \perp}^{\sigma} d}$ ( $j$ is an index of the states in the ferromagnet), $r_{N \rightarrow N}^{\sigma}$ is a scalar reflection coefficient for states incoming from the normal metal, and $r_{F \rightarrow F}^{\sigma}$ is a square matrix describing reflection on the ferromagnetic side. The set of states in the ferromagnet consists of both propagating and evanescent states. The contribution of the latter decreases exponentially with the thickness of the layer.

Concentrating first on the thickness dependence of $g_{\uparrow \mid}^{t}$, we notice that, in view of Eq. (10), the summation in Eq. (5) is carried out over terms containing phase factors $e^{i\left(k_{i \perp}^{\dagger}-k_{\perp \perp}^{\downarrow}\right) d}$. Because of the large differences between majority and minority Fermi surfaces of the ferromagnet, this typically leads to rapidly oscillating terms which mostly cancel out on summing over $\vec{k}_{\|}$. It can be argued ${ }^{19}$ in the spirit of the theory of interlayer exchange coupling ${ }^{39}$ that the only long-range contributions originate from the vicinity of points for which $\nabla_{k_{\|}}\left(k_{i \perp}^{\uparrow}-k_{j \perp}^{\downarrow}\right)=0$, corresponding to the stationary phase of the summand in Eq. (5). These contributions will then exhibit damped oscillations around zero value as seen in Figs. 2 and 4 .

Turning to $g_{\uparrow \downarrow}^{r}$, we find on substituting Eq. (11) into Eq. (4) that there are two thickness-independent contributions. The first comes from summing the $\delta_{n m}$ term in Eq. (4) and is nothing other than the number of states in the normal metal (i.e., the Sharvin conductance). The second comes from the $r_{N \rightarrow N}^{\uparrow} r_{N \rightarrow N}^{l^{*}}$ term and provides an interface-specific correction to the first. Superimposed on these two is the contribution from the thickness-dependent terms which, to lowest order, contain phase factors $e^{i\left(k_{i \perp}^{\sigma}+k_{j \perp}^{\sigma}\right) d}$ and $e^{-i\left(k_{i \perp}^{\sigma}+k_{j \perp}^{\sigma}\right) d}$. Just as in the case of $g_{\uparrow \downarrow}^{t}$, one can argue that the integral over these terms will have oscillatory character. However, the oscillations will have different periods and occur around the constant value set by the first two contributions. It is clear that the value approached asymptotically by $g_{\uparrow \downarrow}^{r}$ is simply the reflection mixing conductance evaluated for a single interface.

The period and damping of oscillations of $g_{\uparrow \downarrow}^{r}$ and $g_{\uparrow \downarrow}^{t}$ as a function of the magnetic-layer thickness $d$ clearly depend (through the $\Lambda^{\sigma}$ ) on the electronic structure of the internal part of the magnetic layer, which for metallic systems is practically identical to that of the bulk material. The amplitudes, on the other hand, are related to the interfacial scattering coefficients introduced in Eqs. (10) and (11). Analyzing the scattering properties of the single interface enables us in the following to understand why the amplitudes of oscillation of $g_{\uparrow \downarrow}^{t}$ are substantially larger than those of $g_{\uparrow \downarrow}^{r}$ for the two systems considered. We begin by noting that the transmission probability for states in the majority-spin channel assumes values close to one over large areas of the Brillouin zone for both $\mathrm{Cu} / \mathrm{Co}$ and $\mathrm{Au} / \mathrm{Fe}$, as illustrated in Fig. 5(a) for the $\mathrm{Cu} / \mathrm{Co}(111)$ interface. For $\mathrm{Cu} / \mathrm{Co}$, this results from the close similarity of the corresponding $\mathrm{Cu}$ and $\mathrm{Co}$ electronic structures. The situation is more complicated for $\mathrm{Au} / \mathrm{Fe}$ because the majority-spin Fermi surface of Fe consists of several sheets, unlike that of Au. However, one of these sheets is made up of states which match well with the states in Au. In the minority-spin channel, on the other hand, the transmission probability varies between 0 and 1; see Fig. 5(b). The maximum sizes of the (absolute value of the) "spin-mixing" products of Eqs. (4) and (5) are therefore determined mostly by the majority-spin scattering coefficients while the modulation, as a function of $\vec{k}_{\|}$, is governed by the corresponding minority-spin coefficients.

The small reflectivity for the majority-spin states has a direct consequence for the values of the mixing conductances. In the case of $g_{\uparrow \downarrow}^{r}$, the second term under the sum in Eq. (4) will typically have a negligible magnitude. This follows directly from $r_{N \rightarrow N}^{\uparrow} \approx 0$ and Eq. (11) and is illustrated in Figs. 5(c) and 5(d) for the $r_{N \rightarrow N}^{\uparrow} r_{N \rightarrow N}^{\jmath^{*}}$ term. As we can see, the only nonzero contributions in this case come from the outer regions of the Brillouin zone, where states from the normal metal are perfectly reflected because of the absence of propagating majority-spin states in the ferromagnet. Independently varying phases (as a function of $\vec{k}_{\|}$) for "up" and "down" reflection coefficients leads, in the course of integration over $\vec{k}_{\|}$, to additional cancellation of already small contributions. The final outcome is that the values of $g_{\uparrow \downarrow}^{r}$ are determined mostly by the first term in the Eq. (4), i.e., the Sharvin conductance of the lead.

Because the interface transmission in the majority-spin channel is uniformly large almost everywhere in the Brillouin zone, the transmission through the magnetic layer also remains large for arbitrary thicknesses, and its magnitude (but not its phase) is only weakly modulated by the multiple scattering within the layer. The magnitude of the $t^{\uparrow} t^{\downarrow^{*}}$ product is then modulated mostly by the variation of the transmission in the minority-spin channel, as a function of $\vec{k}_{\|}$. To demonstrate the effect of the interface scattering on $g_{\uparrow}^{t}$, values of the product $t_{\text {int }}^{\uparrow} t_{\text {int }}^{* *}$ are shown in Figs. 5(e) and 5(f) for a $\mathrm{Cu} / \mathrm{Co}$ (111) interface. Here, $t_{\text {int }}^{\uparrow}$ is defined as the scalar product of the interface transmission vectors: $t_{\text {int }}^{\sigma}$ $=t_{F \rightarrow N}^{\sigma} \cdot t_{N \rightarrow F}^{\sigma}$. As one can see, the values assumed by the real and imaginary parts of this product vary strongly throughout the Brillouin zone. Unlike the case of $g_{\uparrow \downarrow}^{r}$, however, the values span the entire range from -1 to +1 . An imbalance of positive and negative contributions is therefore more likely to produce a sizeable integrated value. The complex values of $t^{\dagger} t^{\downarrow^{*}}$ are further modified by thickness- and $\vec{k}_{\|}$-dependent phase factors discussed above, which leads to the oscillatory 

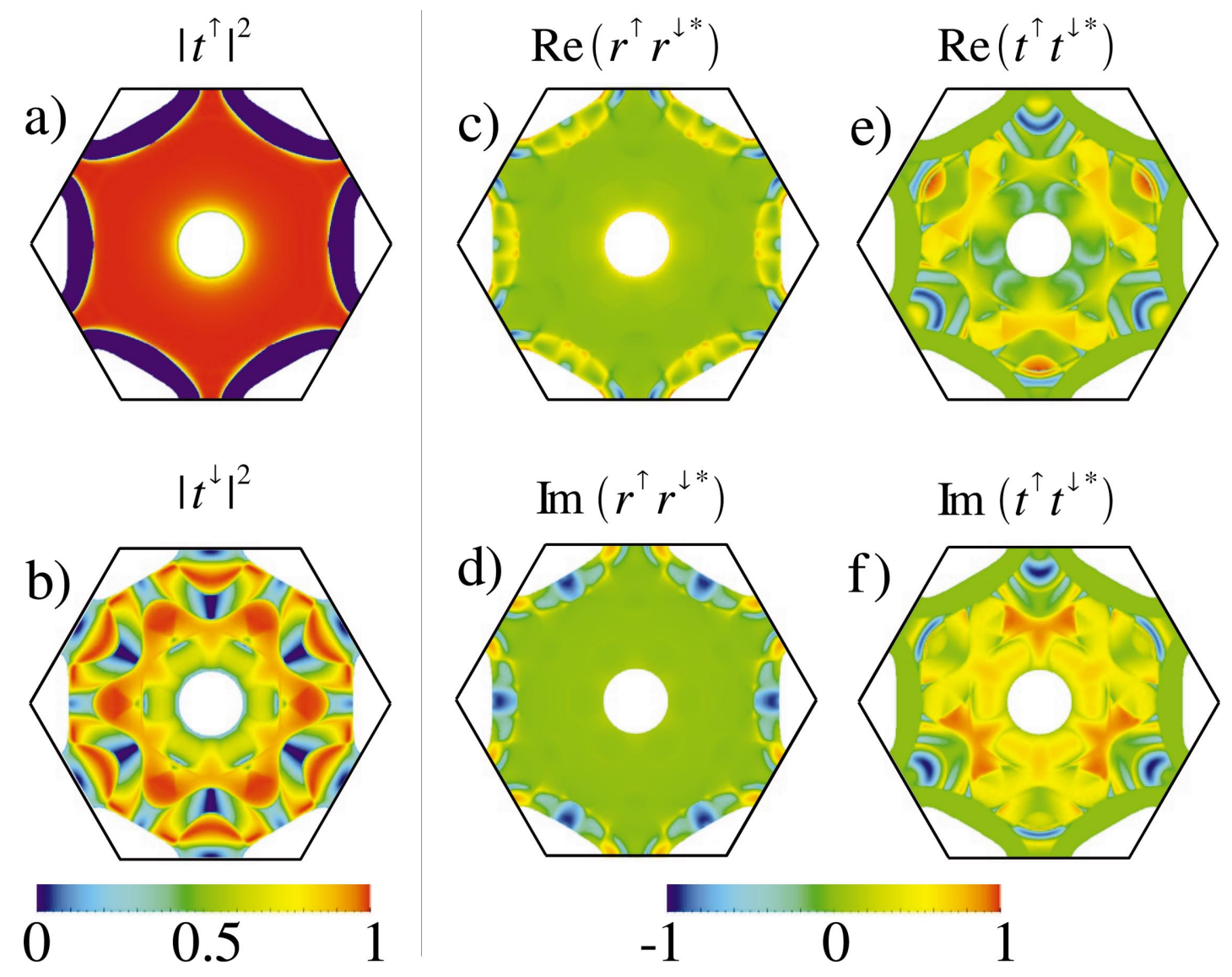

FIG. 5. (Color) Plotted within the first Brillouin zone for the $\mathrm{Cu} / \mathrm{Co}(111)$ interface are transmission probability for (a) majority spins and (b) minority spins. (c) The real and (d) imaginary parts of $r_{N \rightarrow N_{N \rightarrow N}}^{\uparrow} r_{N \rightarrow}^{\downarrow \star}$ (e) The real and (f) imaginary parts of $t_{\text {int }}^{\uparrow} t_{\text {int }}^{\downarrow \star}$ where $t_{\text {int }}^{\sigma}=t_{F \rightarrow N^{*}}^{\sigma} t_{N \rightarrow F}^{\sigma}$ as discussed in the text. Note the different scales for panels (a) and (b) and for (c)-(f).

damping seen in Figs. 2 and 4. We compare the magnitude and damping of these oscillations with those derived from a free-electron model in the Appendix.

Figures 6 and 7 show the same quantities $\left(G_{\uparrow \downarrow}^{r}\right.$ and $\left.G_{\uparrow \downarrow}^{t}\right)$ calculated in the presence of disorder modeled by one monolayer of $50 \%$ alloy added on each side of the magnetic layer. For both systems we have used $10 \times 10$ lateral supercells.
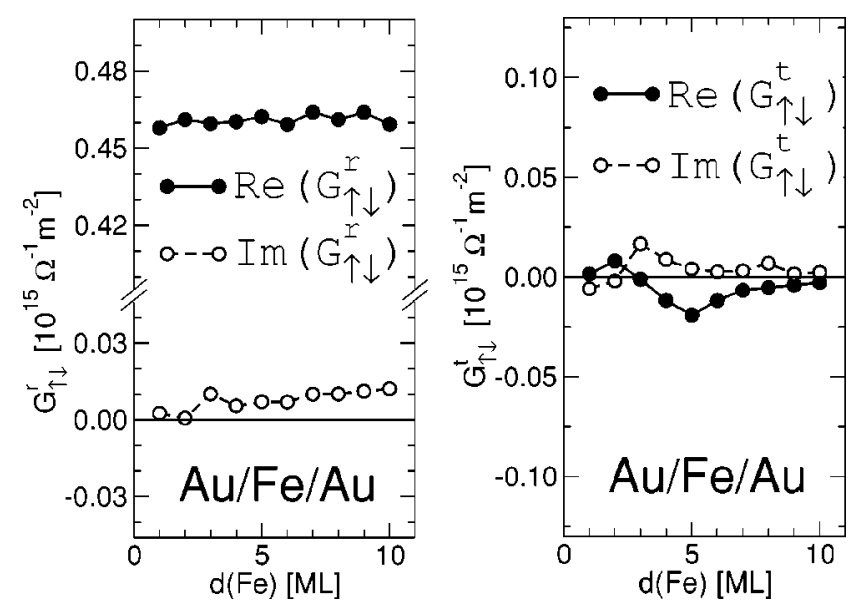

FIG. 6. Spin-mixing conductances of a $\mathrm{Au} / \mathrm{Fe} / \mathrm{Au}(001)$ trilayer with disordered interfaces as a function of the thickness $d$ of the $\mathrm{Fe}$ layer.
The thickness $d$ in this case is that of the clean ferromagnetic layer. For both material systems, the effect of disorder is to strongly reduce the amplitudes of the oscillations. The reflection mixing conductance becomes practically constant at the level of its asymptotic (i.e., interfacial) value. For $G_{\uparrow \downarrow}^{t}$, the oscillations are not entirely damped out but their amplitude is substantially reduced. In fact, the values of $G_{\uparrow \downarrow}^{t}$ become neg-
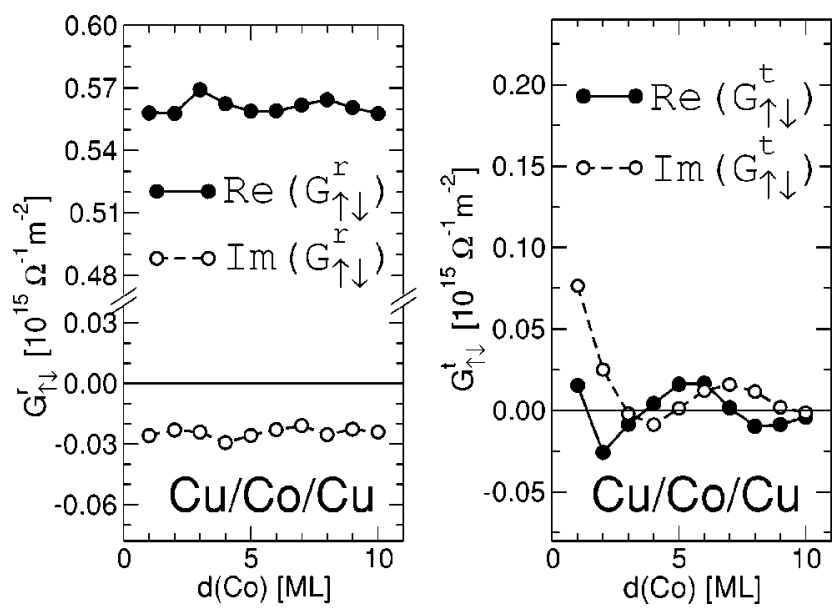

FIG. 7. Spin-mixing conductances of a $\mathrm{Cu} / \mathrm{Co} / \mathrm{Cu}(111)$ trilayer with disordered interfaces as a function of the thickness $d$ of the Co layer. 
TABLE II. Interface conductances in units of $10^{15} \Omega^{-1} m^{-2}$.

\begin{tabular}{lcccccccc}
\hline \hline System & Interface & $G^{\uparrow}$ & $G^{\downarrow}$ & $\operatorname{Re} G_{\uparrow \downarrow}^{r}$ & $\operatorname{Im} G_{\uparrow \downarrow}^{r}$ & $G_{N}^{\text {Sh }}$ & $G_{F \uparrow}^{\text {Sh }}$ & $G_{F \downarrow}^{\text {Sh }}$ \\
\hline $\mathrm{Au} / \mathrm{Fe}$ & clean & 0.40 & 0.08 & 0.466 & 0.005 & 0.46 & 0.83 & 0.46 \\
$(001)$ & alloy & 0.39 & 0.18 & 0.462 & 0.003 & & & \\
$\mathrm{Cu} / \mathrm{Co}$ & clean & 0.42 & 0.38 & 0.546 & 0.015 & 0.58 & 0.46 & 1.08 \\
$(111)$ & alloy & 0.42 & 0.33 & 0.564 & -0.042 & & & \\
\hline \hline
\end{tabular}

ligible compared to $\operatorname{Re} G_{\uparrow \downarrow}^{r}$ for all but the thinnest magnetic layers. In addition, we expect that diffusive scattering in the bulk of the magnetic layer, which for simplicity has not been included here, will have a similar effect.

In view of the above results, we conclude that in a typical situation $A^{\uparrow \downarrow} \approx g_{\uparrow \downarrow}^{r}$, where $g_{\uparrow \downarrow}^{r}$ can be calculated simply for an interface instead of a complete structure. The results of such calculations are listed in Table II for clean and disordered interfaces. The disorder here was modeled by $2 \mathrm{ML}$ of $50 \%$ alloy. In spite of this difference, the values are practically identical to the asymptotic ones seen in Figs. 1, 3, 6, and 7. In particular, $\operatorname{Im} G_{\uparrow \downarrow}^{r}$ assumes values two orders of magnitude smaller than $\operatorname{Re} G_{\uparrow \downarrow}^{r}$, with the latter being close to the Sharvin conductance of the normal metal. This approximate equality results once again from a combination of amplitude (small $\left.\left|r^{\uparrow}\right|\right)$ and uncorrelated spin-up and spin-down phase effects.

The values given in Table II differ somewhat from ones reported previously in Ref. 15. There are two reasons for this. First, the calculations in Ref. 15 were performed using energy-independent muffin-tin orbitals linearized about the centers of gravity of the occupied conduction states. The current implementation ${ }^{34,35}$ uses energy-dependent, (nonlinearized) MTO's, calculated exactly at the Fermi energy which improves the accuracy of the method. Second, on performing the 2D-BZ integration in Eq. (4), it was assumed in Ref. 15 that the contribution to the sum of $\vec{k}_{\|}$points for which there are no propagating states in the ferromagnet should be neglected. However, the lack of propagating states in the ferromagnet does not necessarily prohibit the transfer of spin angular momentum which can be mediated by evanescent states, for example in the case of a magnetic insulator. The contribution from such $\vec{k}_{\|}$points should be included in the 2D-BZ integration.

\section{A. Comparison with experiment}

In Ref. 28, Urban et al. reported room-temperature (RT) observations of increased Gilbert damping for a system consisting of two Fe layers separated by a Au spacer layer. The magnetization of the thinner of the two ferromagnetic layers precesses in the external magnetic field. The other ferromagnetic layer, with the direction of its magnetization fixed, acts as a spin sink. No modification of the damping coefficient was measured for configurations without a second Fe layer. The latter finding is consistent with the prediction given by Eq. (8) in the $\lambda_{\mathrm{SD}} \rightarrow \infty$ limit (well fulfilled for $\mathrm{Au}$ ) as discussed in Sec. II.

In the presence of a second Fe layer, Eq. (9) should be used. Neglecting $\operatorname{Im} \widetilde{A}_{F / N / F}^{\uparrow \downarrow}$ leads to $\gamma_{\text {eff }}=\gamma$ and the damping enhancement

$$
\alpha_{\mathrm{eff}}-\alpha=\frac{\hbar \gamma \operatorname{Re} \tilde{A}_{F / N / F}^{\uparrow \downarrow}}{4 \pi M_{s} d}
$$

where $\alpha \approx 0.0046$ is the damping measured for a single layer. Using ${ }^{40} \gamma=2.1 \mu_{B} / \hbar$ and the values of the interface and Sharvin conductances from Table II (we assume that the values are the same for both $\mathrm{Au} / \mathrm{Fe}$ interfaces), Eq. (12) is compared with the experimental data ${ }^{41}$ in Fig. 8 for various assumptions about $\sigma$ in (9). In the low-temperature limit and neglecting the residual resistivity of the Au layer, $\sigma \rightarrow \infty$, Eq. (12) yields the solid line which is seen to overestimate the damping enhancement compared to the measured results. Using finite values of $\sigma$ will lead to lower values of $\tilde{A}^{\uparrow \downarrow}$ and, indeed, it was found experimentally ${ }^{30}$ that lowering the temperature (increasing the conductivity) increases the damping by as much as about $20 \%$ (open circle in Fig. 8). If we use the room temperature (RT) conductivity due to phonon scattering in crystalline bulk Au, ${ }^{42} \sigma_{\mathrm{ph}}=0.45 \times 10^{8} \Omega^{-1} \mathrm{~m}^{-1}$, the dashed line is obtained which, as expected, is closer to the RT measurements. The agreement with experiment can be further improved by taking into account the possibility of non-negligible residual resistance ${ }^{43}$ of the Au spacer. Assuming, for example, $\sigma_{\text {res }}=0.45 \times 10^{8} \Omega^{-1} \mathrm{~m}^{-1}$ and $\sigma_{\mathrm{ph}}=0$ would

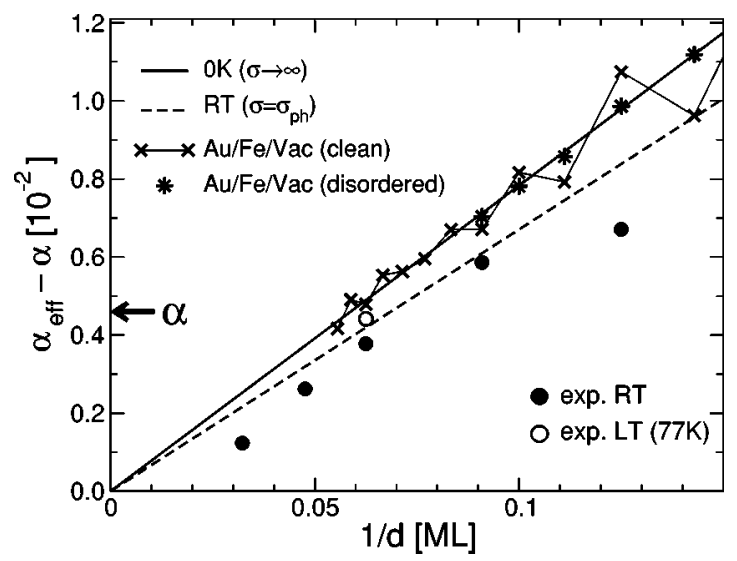

FIG. 8. Enhancement of the Gilbert damping coefficient for an $\mathrm{Fe} / \mathrm{Au} / \mathrm{Fe}$ trilayer as a function of $1 / d$ where $d$ is the thickness of the excited Fe layer. The filled circles $(\bullet)$ are the RT values measured in Ref. 29 and the open one $(\bigcirc)$ is a low temperature value from Ref. 31. The theoretical predictions based on Eq. (12) for $0 \mathrm{~K}$ (with $\sigma \rightarrow \infty$ ) are shown as solid and the RT-corrected (with phonon scattering) ones as dashed lines. The results of $0 \mathrm{~K}$ calculations for a $\mathrm{Au} / \mathrm{Fe} /$ vacuum system are given by crosses $(\times)$ and stars $(*)$ for specular and disordered interfaces, respectively. The value of the Gilbert damping for a single Fe film is marked with an arrow. 
obviously yield the dashed line in the figure while taking $\sigma_{\text {res }}=\sigma_{\mathrm{ph}}=0.45 \times 10^{8} \Omega^{-1} \mathrm{~m}^{-1}$ and $1 / \sigma=1 / \sigma_{\text {res }}+1 / \sigma_{\mathrm{ph}}$ will yield a line very close to the measured points.

The theoretical results represented by the straight lines in Fig. 8 are based upon the asymptotic, single-interface value of $G_{\uparrow \downarrow}^{r}$ from Table II, assuming $G_{\uparrow \downarrow}^{t}$ to be zero. To study possible size-dependent corrections in thin films, the experimental system needs to be represented by a more realistic model than the symmetric $N / F / N$ structures discussed in the previous section. The $\mathrm{Au} / \mathrm{Fe} / \mathrm{GaAs}$ structure used in Ref. 28 differs from these in two important respects. First, the transmission mixing conductance $\left(g_{\uparrow \downarrow}^{t}\right)$ is identically zero because of the insulating substrate. Second, because the reflection is perfect for both spin channels, the thickness-dependent terms in Eq. (11) have larger amplitudes, leading to more pronounced oscillations of $g_{\uparrow !}^{r}$ than those seen in Figs. 1 and 3. To estimate the variation which can result from sizedependent corrections, we have performed a series of calculations for a $\mathrm{Au} / \mathrm{Fe} /$ vacuum structure, using vacuum instead of GaAs for simplicity. The mixing conductance for the other $\mathrm{Au} / \mathrm{Fe}$ interface is kept at its asymptotic value (Table II). The results for perfect (specular) structures, ${ }^{44}$ marked in Fig. 8 with black crosses $(x)$, exhibit oscillations of non-negligible amplitude about the asymptotic values given by the solid line (arbitrarily taking the low-temperature regime, i.e., $\sigma \rightarrow \infty$ for reference). The introduction of interface disorder (two ML of $50 \%-50 \%$ alloy) yields values for the damping [stars $(*)$ in Fig. 8] essentially averaged back to the limit given by the single-interface calculations of Table II.

We have thus demonstrated that direct first-principles calculations can produce values of the damping coefficient in the same range as those measured experimentally. What is more, by taking into account various other sources of scattering in the Au spacer and/or quantum-size effects, the calculations can be brought into very close agreement with experiment. A more definitive quantitative comparison with experiment would require a detailed knowledge of the microscopic structure of the experimental system which is currently not available.

\section{B. Material dependence}

The input parameters of spin pumping theory are scattering matrix elements which are computed using the effective potential of Kohn-Sham theory. This potential is calculated self-consistently and includes electron-electron interaction effects via an exchange-correlation potential approximated using the local spin density approximation, and the Hartree potential. In particular, the modification of interface parameters as a result of magnetic moments being induced in the normal metal by proximity to a ferromagnet (discussed in the Appendix of Ref. 13) is already included in our results in a self-consistent and nonperturbative manner (see Table I). For the $\mathrm{Cu}$ and $\mathrm{Au}$ normal metals we have considered, this effect is small. Expressed in terms of a Stoner enhancement, this is related to the low Fermi level densities of states, $D\left(\varepsilon_{F}\right)$, of these metals. Viewing it in this way poses the question of the possibility of finding much larger effects for materials such as Pd and Pt which have a large density of states at the Fermi
TABLE III. Density of states at the Fermi level, Stoner enhancement factor, and typical Sharvin conductances for bulk fcc $\mathrm{Cu}, \mathrm{Pd}$ and $\mathrm{Pt}$ and bcc Ta. Typical values of the Stoner parameter, $I_{x c}$, were taken from Refs. 45 and 46.

\begin{tabular}{clccc}
\hline \hline & $\mathrm{Cu}$ & $\mathrm{Ta}$ & $\mathrm{Pd}$ & $\mathrm{Pt}$ \\
\hline$D\left(\varepsilon_{F}\right)[$ states/(Ry atom spin) $]$ & 2 & 10 & 15 & 12 \\
{$\left[1-D\left(\varepsilon_{F}\right) I_{x c}\right]^{-1}$} & 1.1 & 1.9 & 4.4 & 2.2 \\
$G^{S h}\left(10^{15} \Omega^{-1} \mathrm{~m}^{-2}\right)$ & 0.58 & 0.97 & 0.62 & 0.68 \\
\hline \hline
\end{tabular}

level (see Table III) and are known to be close to a ferromagnetic transition as expressed by the susceptibility enhancement $\chi^{\prime} \chi_{0}=\left[1-D\left(\varepsilon_{F}\right) I_{x c}\right]^{-1}$, also included in the table. To calculate this factor, typical values of the Stoner parameter, $I_{x c}$, were taken from Refs. 45 and 46.

To examine whether enhancements of the Gilbert damping parameter recently reported $\mathrm{d}^{47,48}$ for thin layers of $\mathrm{Ta}, \mathrm{Pd}$ and $\mathrm{Pt}$ compared to $\mathrm{Cu}$ are related to their large Fermi level densities of states, we need to reexamine how the electronic structure enters our description of the Gilbert damping. In the spin-pumping formulation, the quantities determining the damping enhancement are not densities of states but transmission and reflection mixing conductances determined from the scattering matrix. In most situations, we have seen, the former is negligible and the latter reduces to its real part, $\operatorname{Re} G_{\uparrow \downarrow}^{r}$, which in turn is very close to the Sharvin conductance of the normal metal. Values of this quantity are given for $\mathrm{Cu}, \mathrm{Ta}, \mathrm{Pd}$, and $\mathrm{Pt}$ in the last row of the Table. It is seen that the Sharvin conductance changes less than $D\left(\varepsilon_{F}\right)$. More significantly, with a maximum for $\mathrm{Ta}$, the trend does not correspond to that observed experimentally: ${ }^{47,48} \mathrm{Cu} \rightarrow \mathrm{Ta}$ $\rightarrow \mathrm{Pd} \rightarrow \mathrm{Pt}$. We believe that the explanation should be sought elsewhere, possibly in the increasing spin-orbit interaction which will lead to the heavier materials behaving as more efficient spin sinks. ${ }^{4,5}$ To examine this suggestion in detail from first principles requires a formulation of the theory to which the spin-orbit interaction can be readily added.

\section{Spin-current-induced torque}

The mixing conductances calculated above, which describe how a spin current flows through the system in response to an externally applied spin accumulation $\boldsymbol{\mu}$ [defined as a vector with length equal to half of the spin-splitting of the chemical potentials $\left.|\boldsymbol{\mu}|=\left(\mu_{\uparrow}-\mu_{\downarrow}\right) / 2\right]$, also describe the spin torque exerted on the moment of the magnetic layer (see e.g., Refs. 15, 17-19, and 22). Consider, for example, the situation where the spin accumulation has been induced by some means in the left lead only and the ferromagnet is magnetized along the $z$ axis. The spin current incident on the interface is proportional to the number of incoming channels in the lead $\mathbf{I}_{\text {in }}^{\mathrm{L}}=(1 / 2 \pi) g_{N}^{\mathrm{Sh}} \boldsymbol{\mu}$ whereas the transmitted spin current is given by ${ }^{22}$

$$
\mathbf{I}_{\text {out }}^{\mathrm{R}}=\frac{1}{2 \pi}\left(\begin{array}{ccc}
\operatorname{Re} g_{\uparrow \downarrow}^{t} & \operatorname{Im} g_{\uparrow \downarrow}^{t} & 0 \\
-\operatorname{Im} g_{\uparrow \downarrow}^{t} & \operatorname{Re} g_{\uparrow \downarrow}^{t} & 0 \\
0 & 0 & \frac{g^{\uparrow}+g^{\downarrow}}{2}
\end{array}\right) \boldsymbol{\mu}
$$

and the reflected spin current by 


$$
\mathbf{I}_{\mathrm{out}}^{\mathrm{L}}=\frac{1}{2 \pi}\left(\begin{array}{ccc}
g_{\mathrm{N}}^{\mathrm{Sh}}-\operatorname{Re} g_{\uparrow \downarrow}^{r} & -\operatorname{Im} g_{\uparrow \downarrow}^{r} & 0 \\
\operatorname{Im} g_{\uparrow \downarrow}^{r} & g_{\mathrm{N}}^{\mathrm{Sh}}-\operatorname{Re} g_{\uparrow \downarrow}^{r} & 0 \\
0 & 0 & g_{\mathrm{N}}^{\mathrm{Sh}}-\frac{g^{\uparrow}+g^{\downarrow}}{2}
\end{array}\right) \boldsymbol{\mu}
$$

where $g^{\sigma}=\Sigma_{n m}\left|t_{n m}^{\sigma}\right|^{2}$ are the conventional Landauer-Büttiker conductances. Therefore $g_{\uparrow}^{t}$ determines the transverse component of the transmitted spin current subject to precession and absorption within the magnetic layer. Similarly, the real and imaginary parts of $g_{N}^{\mathrm{Sh}}-g_{\uparrow \downarrow}^{r}=\Sigma_{m n} r_{m n}^{\uparrow} r_{m n}^{\downarrow \star}$ are related to the components of the reflected transverse spin current. The rapid decay of $g_{\uparrow \downarrow}^{t}$ (and $g_{N}^{\mathrm{Sh}}-g_{\uparrow \downarrow}^{r}$ ) discussed in previous paragraphs as a function of increasing magnetic layer thickness implies that the absorption of the transverse component of the spin current occurs within a few monolayers of the $N / F$ interface. In particular we find that the presence of the disorder improves the effectiveness of the absorption. The limit $g_{\uparrow \downarrow}^{t} \rightarrow 0$ and $g_{\uparrow \downarrow}^{r} \rightarrow g_{N}^{\text {Sh }}$ corresponds to the situation where all of the incoming transverse polarized spin current is absorbed in the magnetic layer. The torque is then proportional to the Sharvin conductance of the normal metal. As demonstrated in Figs. 1-4, 6, and 7 this is the situation for all but the thinnest (few monolayers) and cleanest magnetic layers.

\section{CONCLUSIONS}

In summary, we have calculated the transmission and reflection mixing conductances that govern the nonlocal effects in the ferromagnetic magnetization dynamics for two commonly used $N / F$ combinations: $\mathrm{Au} / \mathrm{Fe}$ and $\mathrm{Cu} / \mathrm{Co}$. In both cases, the transmission mixing conductance $g_{\uparrow \downarrow}^{t}$ is much smaller than the reflection mixing conductance $g_{\uparrow \downarrow}^{r}$, except for the thinnest magnetic films, only a few atoms thick. Even for such thin films, $g_{\uparrow \downarrow}^{t}$ is smaller than $g_{\uparrow \downarrow}^{r}$. Furthermore, $g_{\uparrow \downarrow}^{t}$ is more sensitive to disorder, even a small amount of which reduces it to zero while having only a small effect on $g_{\uparrow \downarrow}^{r}$ as shown in Figs. 6 and 7. For all thicknesses, $\operatorname{Re} g_{\uparrow \downarrow}^{r} \gg \operatorname{Im} g_{\uparrow \downarrow}^{r}$ and $\operatorname{Re} g_{\uparrow}^{r}$ is very close to its interfacial value (i.e., the mixing conductance of the infinitely thick magnetic film). The general formulas (6) and (7) predict that the spin pumping renormalizes both the Gilbert damping $(\alpha)$ and the gyromagnetic ratio $(\gamma)$ of a ferromagnetic film embedded in a conducting nonmagnetic medium. However, in view of the results discussed in the previous section, we conclude that, for all but the thinnest and cleanest magnetic layers, the only effect of the spin pumping is to enhance the Gilbert damping. The correction is directly proportional to the real part of the reflection mixing conductance and is essentially an interface property. We also find that oscillatory effects are averaged out for realistic band structures, especially in the presence of disorder. $\operatorname{Re} g_{\uparrow \downarrow}^{r}$ (which determines the damping enhancement of a single ferromagnetic film embedded in a perfect spinsink medium) is usually very close to $g_{N}^{\text {Sh }}$ for intermetallic interfaces ${ }^{15,19}$ [being in general bounded by $2 g_{N}^{\mathrm{Sh}}$ according to its definition, Eq. (4)]. These results also apply to the spin-current-induced magnetization reversal in intermetallic systems, indicating that the "effective field" correction due to

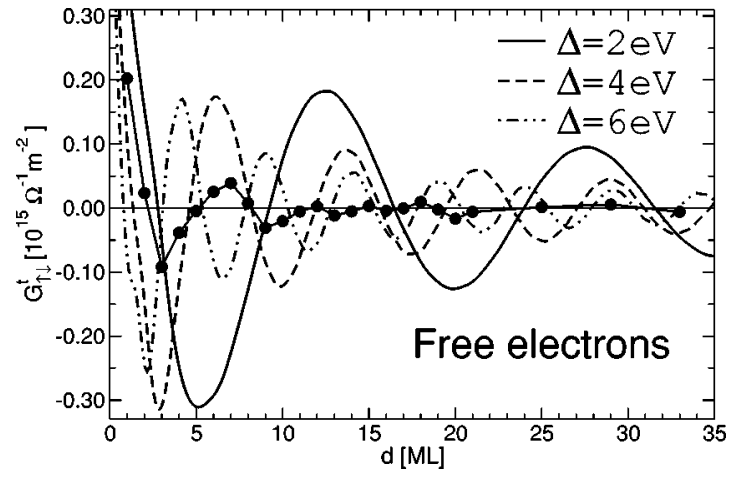

FIG. 9. The real part of $G_{\uparrow \downarrow}^{t}$ calculated for a free electron model with $\varepsilon_{F}=7 \mathrm{eV}$ (energy measured from the bottom of the parabolic conduction band in the normal metal) and various choices of the exchange splitting $\Delta$. The interlayer distance is taken to be the same as for the $\mathrm{Cu} / \mathrm{Co}(111)$ system. The results of the first-principles calculations (๑) from Fig. 4 are included for comparison.

the imaginary part of the mixing conductance and bulk contributions to the torque are very small.

\section{ACKNOWLEDGMENTS}

This work is part of the research program for the "Stichting voor Fundamenteel Onderzoek der Materie" (FOM) and the use of supercomputer facilities was sponsored by the "Stichting Nationale Computer Faciliteiten" (NCF), both financially supported by the "Nederlandse Organisatie voor Wetenschappelijk Onderzoek" (NWO). It was also supported by the European Commission's RT Network Computational Magnetoelectronics (Contract No. HPRN-CT-2000-00143), by the NEDO International Joint Research Grant Program Nano-Magnetoelectronics, and by The Harvard Society of Fellows. M.Z. wishes also to acknowledge support from KBN Grant No. PBZ-KBN-044/P03-2001.

\section{APPENDIX: COMPARISON WITH A FREE-ELECTRON MODEL}

A combination of interfacial and bulk dephasing mechanisms, discussed in Sec. IV, ensures that in the asymptotic (thick magnetic layer) limit the spin-pumping mixingconductance, $A^{\uparrow \downarrow}$, reduces to the reflection mixing conductance $g_{\uparrow \downarrow}^{r}$, with the latter quantity assuming values which are predominantly real and equal to those determined for a single $N / F$ interface. Thinner layers exhibit oscillatory behavior which is most pronounced for $G_{\uparrow \downarrow}^{t}$ (Figs. 2 and 4). The amplitude of oscillation, however, is at most $20 \%$ of the asymptotic value of $G_{\uparrow \downarrow}^{r}$ and decreases to less than $5 \%$ for layers more than $10 \mathrm{ML}$ thick. This fast decay, found even for clean, fully coherent structures, contrasts with results reported in Ref. 14 for a free-electron model. For thin layers, Mills found the damping coefficient oscillated with amplitude in the range of $80 \%$ of the asymptotic value and, for layers several tens of MLs thick, it was still of order $10 \%$. This feature of the free-electron model is illustrated vividly in Fig. 9 by comparing $\operatorname{Re}\left(G_{\uparrow\rfloor}^{t}\right)$ for $\mathrm{Cu} / \mathrm{Co} / \mathrm{Cu}(111)$ from Fig. 4 with the corresponding results calculated for free electrons. 
In our free-electron calculation, the Fermi energy in the nonmagnetic material was taken to be $7 \mathrm{eV}$ in order to obtain the correct value for the Sharvin conductance of $\mathrm{Cu}$ and the effect of changing the exchange splitting $\Delta$ of the ferromagnet was studied. For $\Delta=2,4,6 \mathrm{eV}$, the amplitude of oscillation is much larger and the decay is much slower than what we find for the more realistic multi-band electronic structures. As might be expected, increasing the exchange-splitting from 2 to $6 \mathrm{eV}$ leads to a shorter period and more rapid decay of the oscillations. However, in order to mimic the parameter-free result, an exchange splitting in the range of $10 \mathrm{eV}$ would be needed (not shown in the figure). Such a large value cannot be justified either on theoretical or experimental grounds. This discrepancy illustrates the difficulty of mapping the complex electronic structure of transition metals onto single band models in a meaningful way. Free-electron models do not adequately describe the effectiveness of the thicknessdependent "bulk" dephasing in the ferromagnet. What is more, they also cannot reproduce the complex spin- and $\vec{k}_{\|}$-dependence of the interface scattering coefficients (illustrated in Fig. 5) which results from the mismatch of the normal metal electronic structure and the quite different majority- and minority-spin electronic structures of a ferromagnetic metal. For single band free-electron models, the interface scattering coefficients contain much less structure and consequently this model fails to take into account even qualitatively the dephasing effect of the interface.
*Permanent address: Institute of Molecular Physics, P.A.N., Smoluchowskiego 17, 60-179 Poznań, Poland.

${ }^{1}$ T. L. Gilbert, Phys. Rev. 100, 1243 (1955); L. D. Landau, E. M. Lifshitz, and L. P. Pitaevski, Statistical Physics, part 2, 3rd ed. (Pergamon, Oxford, 1980).

${ }^{2}$ R. Arias and D. L. Mills, Phys. Rev. B 60, 7395 (1999).

${ }^{3}$ W. Platow, A. N. Anisimov, G. L. Dunifer, M. Farle, and K. Baberschke, Phys. Rev. B 58, 5611 (1998).

${ }^{4}$ Y. Tserkovnyak, A. Brataas, and G. E. W. Bauer, Phys. Rev. Lett. 88, 117601 (2002).

${ }^{5}$ Y. Tserkovnyak, A. Brataas, and G. E. W. Bauer, Phys. Rev. B 66, 224403 (2002).

${ }^{6}$ L. Berger, Phys. Rev. B 54, 9353 (1996).

${ }^{7}$ A. Brataas, Y. Tserkovnyak, G. E. W. Bauer, and B. I. Halperin, Phys. Rev. B 66, 060404(R) (2002).

${ }^{8}$ B. Heinrich, Y. Tserkovnyak, G. Woltersdorf, A. Brataas, R. Urban, and G. E. W. Bauer, Phys. Rev. Lett. 90, 187601 (2003).

${ }^{9}$ Y. Tserkovnyak, A. Brataas, and G. E. W. Bauer, J. Appl. Phys. 93, 7534 (2003).

${ }^{10}$ Y. Tserkovnyak, A. Brataas, and G. E. W. Bauer, Phys. Rev. B 67, 140404(R) (2003).

${ }^{11}$ R. H. Silsbee, A. Janossy, and P. Monod, Phys. Rev. B 19, 4382 (1979).

${ }^{12}$ E. Šimánek and B. Heinrich, Phys. Rev. B 67, 144418 (2003).

${ }^{13}$ E. Šimánek, Phys. Rev. B 68, 224403 (2003).

${ }^{14}$ D. L. Mills, Phys. Rev. B 68, 014419 (2003).

${ }^{15}$ K. Xia, P. J. Kelly, G. E. W. Bauer, A. Brataas, and I. Turek, Phys. Rev. B 65, 220401(R) (2002).

${ }^{16}$ S. I. Kiselev, J. C. Sankey, I. N. Krivorotov, N. C. Emley, R. J. Schoelkopf, R. A. Buhrman, and D. C. Ralph, Nature (London) 425, 380 (2003) and references therein.

${ }^{17}$ J. C. Slonczewski, J. Magn. Magn. Mater. 159, L1 (1996); X. Waintal, E. B. Myers, P. W. Brouwer, and D. C. Ralph, Phys. Rev. B 62, 12317 (2000).

${ }^{18}$ A. Brataas, Y. V. Nazarov, and G. E. W. Bauer, Phys. Rev. Lett. 84, 2481 (2000).

${ }^{19}$ M. D. Stiles and A. Zangwill, Phys. Rev. B 66, 014407 (2002).

${ }^{20}$ E. B. Myers, D. C. Ralph, J. A. Katine, R. N. Louie, and R. A. Buhrman, Science 285, 867 (1999).

${ }^{21}$ Note that there is a sign error in Refs. 4 and 5 the quantity $A_{i}$ defined in Eq. (6) of Ref. 4 is equal to $-\operatorname{Im} A^{\uparrow \downarrow}$, not $\operatorname{Im} A^{\uparrow \downarrow}$.
${ }^{22}$ A. Brataas, G. Zárand, Y. Tserkovnyak, and G. E. W. Bauer, Phys. Rev. Lett. 91, 166601 (2003).

${ }^{23}$ S. Datta, Electronic Transport in Mesoscopic Systems, (Cambridge University Press, Cambridge, 1995).

${ }^{24}$ The renormalization of the mixing conductances has a close analogy in the discussion of various ways of measuring the resistance in mesoscopic systems. It is argued (see, e.g., Ref. 23) that for the standard conductances, the "bare" quantities $g$ $=\Sigma_{m n}\left|t_{m n}\right|^{2}$ are suitable for the description of two-point measurements with the reservoirs acting as both the current and voltage contacts. For an idealized four-point measurements with the voltage drop being measured directly across the scatterer (e.g., an interface, as in our case) the renormalized conductance (with the contact resistance subtracted) is needed. In the simplest limit of a single-mode conductor, with $g^{\mathrm{Sh}}=1$, the renormalized conductance reduces to the "old" Landauer formula $\widetilde{g}=g /(1-g)$. It is intuitively clear that these are also the quantities that one needs to use when the scatterer is embedded in the diffuse environment.

${ }^{25}$ G. E. W. Bauer, Y. Tserkovnyak, D. Huertas-Hernando, and A. Brataas, Phys. Rev. B 67, 094421 (2003).

${ }^{26}$ K. Xia, P. J. Kelly, G. E. W. Bauer, I. Turek, J. Kudrnovský, and V. Drchal, Phys. Rev. B 63, 064407 (2001).

${ }^{27}$ K. Xia, P. J. Kelly, G. E. W. Bauer, and I. Turek, Phys. Rev. Lett. 89, 166603 (2002).

${ }^{28}$ R. Urban, G. Woltersdorf, and B. Heinrich, Phys. Rev. Lett. 87, 217204 (2001).

${ }^{29}$ B. Heinrich, R. Urban, and G. Woltersdorf, J. Appl. Phys. 91, 7523 (2002).

${ }^{30}$ B. Heinrich , G. Woltersdorf, R. Urban, and E. Šimánek, J. Appl. Phys. 93, 7545 (2003).

${ }^{31}$ J. A. Katine, F. J. Albert, R. A. Buhrman, E. B. Myers, and D. C. Ralph, Phys. Rev. Lett. 84, 3149 (2000).

${ }^{32}$ I. Turek, V. Drchal, J. Kudrnovský, M. Šob, and P. Weinberger, Electronic Structure of Disordered Alloys, Surfaces and Interfaces (Kluwer, Boston, 1997); O. K. Andersen, O. Jepsen, and D. Glötzel, in Highlights in Condensed Matter Theory, edited by F. Bassani, F. Fumi, and M. P. Tosi (North-Holland, Amsterdam, 1985), p.59.

${ }^{33}$ J. P. Perdew and A. Zunger, Phys. Rev. B 23, 5048 (1981)

${ }^{34}$ M. Zwierzycki, K. Xia, P. J. Kelly, G. E. W. Bauer, and I. Turek, 
Phys. Rev. B 67, 092401 (2003).

${ }^{35} \mathrm{~K}$. Xia et al. (unpublished).

${ }^{36}$ J-T. Wang, Z-Q. Li, Q. Sun, and Y. Kawazoe, J. Magn. Magn. Mater. 183, 42 (1998)

${ }^{37}$ M. G. Samant, J. Stöhr, S. S. P. Parkin, G. A. Held, B. D. Hermsmeier, F. Herman, M. van Schilfgaarde, L.-C. Duda, D. C. Mancini, N. Wassdahl, and R. Nakajima, Phys. Rev. Lett. 72, 1112 (1994)

${ }^{38}$ S. Krompiewski, F. Süss, B. Zellerman, and U. Krey, J. Magn. Magn. Mater. 148, 198 (1995).

${ }^{39}$ P. Bruno, Phys. Rev. B 52, 411 (1995).

${ }^{40}$ B. Heinrich, K. B. Urquhart, A. S. Arrott, J. F. Cochran, K. Myrtle, and S. T. Purcell, Phys. Rev. Lett. 59, 1756 (1987).

${ }^{41}$ The data reported in Refs. 28 and 30 were underestimated by a factor 1.15 (B. Heinrich, private communication). Here we use corrected values.

${ }^{42}$ http://www.webelements.com
${ }^{43}$ Measurements of the sheet conductivity (Ref. 30) indicate that the $\mathrm{Au}$ layers used in the experiments may have non-negligible residual resistances. The conductivity entering Eq. (9) should not include interfacial-scattering contributions so the measurement of Ref. 30 cannot be used directly to obtain $\sigma_{\text {res }}$.

${ }^{44}$ It should be stressed, however, that although this calculation might give an estimate of the quantum-size effects, the applicability of Eq. (9) requires that the system be globally diffuse (Ref. 25 ). The treatment of Ref. 25 would need to be generalized to enable consideration of nearly specular structures with little bulk disorder.

${ }^{45}$ O. Gunnarsson, J. Phys. F: Met. Phys. 6, 587 (1976).

${ }^{46}$ J. F. Janak, Phys. Rev. B 16, 255 (1977).

${ }^{47}$ S. Mizukami, Y. Ando, and T. Miyazaki, Jpn. J. Appl. Phys., Part 1 40, 580 (2001).

${ }^{48}$ S. Mizukami, Y. Ando, and T. Miyazaki, J. Magn. Magn. Mater. 226-230, 1640 (2001). 